CHRONIQUE DE LA RECHERCHE

\title{
LE RENOUVEAU DES ETUDES EINSTEINIENNES
}

1979, l'année du centenaire de la naissance d'Einstein, fut l'occasion, dans le monde entier, de nombreuses manifestations et célébrations. Ce fut aussi l'année du renouveau des études que l'on pourrait - paraphrasant Koyré qualifier d'einsteiniennes. Tout se passe comme si le mythe qui entourait la personnalité d'Einstein et qui, paradoxalement, avait empêché de prendre au sérieux son cuvre pour en faire un objet d'étude, avait été exorcisé par tant de cérémonies officielles : Einstein, de demi-dieu qu'il était brusquement devenu un jour de 1919, à l'annonce de la vérification de sa théorie de la relativité générale par la déviation des rayons lumineux observée par Eddington, redevenait un homme. En caricaturant à peine, on peut dire que l'évolution intellectuelle d'Einstein, tenue jusqu'alors pour tout aussi peu analysable que celle de JésusChrist, s'était brusquement révélée digne d'intérêt historique.

Où en sont, neuf ans après, ces études einsteiniennes et qu'ont-elles produit ? C'est ce que je me propose d'analyser ici, sans prétendre à l'exhaustivité, bien au contraire. Ces études peuvent, schématiquement, être regroupées sous quatre rubriques. D'abord une série de recueils rassemblant les contributions aux différents colloques du Centenaire tenus durant l'année 1979 et dont on trouvera la liste dans la bibliographie ci-dessous. Ensuite, un ensemble d'articles parus dans les diverses revues d'histoire des sciences (anglo-saxonnes essentiellement) dont certains ont d'ores et déjà été regroupés par leurs auteurs en volumes. Citons, entre autres, les "Jefferson Lectures" de G. Holton publiées sous le titre The Advancement of Science and its Burdens, The Shaky Game, Einstein Realism and the Quantum Theory par A. Fine, The Young Einstein par L. Pyenson... Viennent ensuite deux livres entièrement consacrés à l'œuvre scientifique d'Einstein : par A. Miller d'une part, Special Theory of Relativity (19051911), qui depuis sa publication en 1981 reste sans conteste la réference pour qui veut comprendre les origines de la théorie de la relativité restreinte et ce qui la différencie des autres recherches théoriques de l'époque, notamment celles de Poincare ; et d'autre part, Subtle is the Lord... The Science and the Life of Albert Einstein, biographie scientifique écrite par l'un des plus grands physiciens de ce temps qui, de ce fait, n'est pas d'une lecture facile pour les non-physiciens, mais représente une tentative d'histoire de la physique par un

Revue de synthèse: IV $\mathrm{S}$. $\mathrm{N}^{\circ} 2$, avril-juin 1988. 
physicien à destination des physiciens sans équivalent : on rêve d'une époque où la lecture de ce livre ferait partie intégrante du cursus des études de physique fondamentale!

Vient enfin, et ce n'est pas l'un des moindres acquis de ces huit dernières années, le début de la publication de l'édition complète et critique des écrits d'Einstein - dont le premier volume est paru récemment - éditée (au sens américain du terme) par une équipe de chercheurs de Boston, sous la direction de J. Stachel, et publiée chez Princeton University Press.

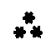

A vrai dire, l'idée d'une publication des œuvres complètes d'Einstein ne date pas de 1979. Dès 1956, Robert Oppenheimer, alors directeur de l'Institute for Advanced Study de Princeton (où Einstein avait trouvé refuge dès 1933 et où il vécut jusqu'à sa mort, en 1955), avait envisagé la publication des œuvres scientifiques. Cette proposition n'avait cependant pas rencontré l'assentiment d'Helen Dukas qui assurait depuis 1928 le secrétariat d'Einstein; Helen Dukas pensait que l'ensemble de l'œuvre d'Einstein (écrits scientifiques bien sûr, mais aussi écrits philosophiques, politiques, épistémologiques, sans compter l'énorme correspondance) méritait une publication intégrale et qu'il ne convenait pas de faire un sort spécial aux articles scientifiques. De 1955 à 1982, date de sa mort, H. Dukas accomplit un travail d'archivage absolument gigantesque, retrouvant les lettres perdues là où elles se trouvaient dispersées dans les familles et les institutions, mettant en ordre l'impressionnant corpus de documents (manuscrits, papiers officiels et autres) laissés par Einstein. Notons à ce propos, que s’il est vrai qu'Einstein dut s'enfuir d'Allemagne en 1933 (il se trouvait en Angleterre au moment de la prise de pouvoir par Hitler et ne put rentrer en Allemagne), il n'en reste pas moins que ses papiers et ses meubles purent quand même lui être expédiés aux États-Unis, grâce au dévouement de certains de ses amis.

Grâce à $H$. Dukas (et $O$. Nathan, légataire universel d'Einstein), le fonds d'archives dont disposent aujourd'hui les chercheurs comporte plus de mille documents non épistolaires et treize cent lettres, le tout dans un état d'ordre exceptionnel. L'original des archives se trouve à l'Université Hébraïque de Jérusalem à qui Einstein avait légué ses droits et l'on dispose de deux (et bientôt trois) copies, à Princeton et Boston. C'est à partir de ces copies que travaille l'équipe qui, sous la direction de J. Stachel, prépare l'édition (en plus de trente volumes!) des auvres complètes et de la correspondance d'Einstein, assortie de nombreux documents permettant de mieux comprendre l'homme et ses idées. Cette édition, bien que publiée aux États-Unis, est en langue originale, c'est-à-dire à quatre-vingt-quinze pour cent en allemand (les notes, toutefois, sont rédigées en anglais). Elle procède par ordre chronologique; aussi le premier volume, paru l'année dernière, s'intitule-t-il The Early Years 1879-1902.

Pour comprendre les raisons de cette périodisation, et plus précisément du choix de la date de 1902, il convient de rappeler que c'est en 1902 qu'Einstein, après plusieurs années de chômage, entre au bureau des brevets de Berne et commence à gagner sa vie. C'est en tant qu'employé de cette administration 
qu'il rédigera et publiera, en 1905 , véritable " annus mirabilis », cinq articles qui tous, à des titres divers, devaient infléchir, voire révolutionner, le cours de la physique.

C'est donc un véritable « Bildungsroman " que nous livrent aujourd'hui les éditeurs. Ce n'est d'ailleurs pas l'un des moindres intérêts de cet ouvrage que de donner à lire l'histoire d'un jeune homme ordinaire nommé Albert Einstein. Chômage, échec aux examens, solitude, déception sentimentale, chantage affectif des parents, culpabilité face à l'incapacité de leur venir en aide, misère et contraintes sociales d'un milieu somme toute étriqué (celui des juifs assimilés de Bavière), Einstein a connu tout cela... comme la plupart des jeunes gens. Force est de constater que vingt ans n'est pas le plus bel âge de la vie, ce que l'on savait déjà depuis que Nizan a osé le proclamer.

Ce que l'on ne savait pas, en revanche, et que révèle un ensemble de lettres récemment découvertes par l'équipe de J. Stachel, lettres écrites par Einstein à sa première femme Mileva Marić au temps où ils n'étaient pas encore mariés, c'est le rôle capital qu'a joué cette femme dans le développement des idées scientifiques d'Einstein, dans les années qui ont précédé l'éclosion tant célébrée de 1905. Mileva Marić, jeune fille d'origine serbe, fille d'un officier de l'administration hongroise, fait partie de cette génération de jeunes " orientales " qui, telle Marie Curie, sont venues étudier les sciences en Europe occidentale; ce qui fut pour elles le moyen d'échapper à la fois au sous-développement culturel et à l'avenir de femmes soumises qu'on leur réservait. En quoi d'ailleurs, ces jeunes filles se trouvaient finalement en meilleure position d'émancipation que leurs contemporaines anglaises, françaises ou allemandes. C'est à l'ETH (célèbre école polytechnique de Zürich) qu'Einstein et Mileva Marić se sont connus. La correspondance publiée ici, outre qu'elle nous montre un Einstein inconnu, normalement amoureux, avec toute l'ardeur que l'on attend d'un jeune homme romantique et sensible, présente l'intérêt unique de nous révéler les étapes de leur collaboration scientifique, éclairant d'un jour nouveau les années de formation d'Einstein.

Ainsi pourra-t-on constater que dès septembre 1899 (six ans donc avant l'article fondateur de la relativité restreinte), Einstein, commentant l'usage fait par Helmoltz du principe de moindre action, écrit à Mileva : " Je suis de plus en plus persuadé que l'électrodynamique des corps en mouvement, telle qu'elle est présentée à l'heure actuelle, ne correspond pas à la réalité [Wirklichkeit] et que l'on doit pouvoir la représenter de façon plus simple. Je crois, ajoute-t-il, que les forces électriques ne peuvent être définies que dans l'espace vide " (et donc sans le secours de l'éther). Ceux qui sont familiers avec l'article de 1905 auront reconnu là les prémisses et les conclusions de cet article. Et Einstein de conclure : «Lequel des deux points de vue adopter [celui de Helmoltz ou le sien encore non entièrement élaborél, c'est ce que nous montrera l'étude du rayonnement " (souligné par moi). Si l'on songe qu'en 1905 Einstein a produit, à quelques mois d'intervalles, l'article sur la relativité restreinte dont il vient d'être question et un autre article non moins " génial " portant sur la nature discontinue du rayonnement et apparemment sans relation avec le précédent, on conçoit l'importance que peuvent avoir pour l'histoire des idées scientifiques des indications de ce type, soulignant l'unité des deux problèmes. 
On verra également que, dès 1899, Einstein (et Mileva dont c'était plus ou moins le sujet de thèse) avaient comme projet d'établir, sur le modèle de la théorie cinétique et statistique des gaz élaborée par Boltzmann, un parallèle entre l'agitation thermique des atomes d'un solide (autrement dit sa chaleur spécifique) et le spectre de la lumière émise par ce même solide. Or l'article de 1905 concluant à la nécessité d'une quantification du rayonnement repose précisément sur une analogie (de forme, mais qu'Einstein décide de prendre au sérieux) entre l'entropie du rayonnement et celle d'un gaz « à la Boltzmann ». Plus même : Einstein, en 1907, dans un article traitant de la chaleur spécifique des solides, étend la quantification, jusqu'alors considérée comme une propriété du rayonnement, au mouvement des atomes d'un solide. On voit que les idées, sur lesquelles devait s'élever dans les trente premières années du siècle la théorie quantique, étaient déjà en germe dans les discussions qu'avaient Mileva et Einstein en 1899.

La publication de la correspondance entre Einstein et Mileva Marić réserve d'autres surprises à l'historien des sciences. En particulier, le fait, ignoré jusqu'alors, qu'Einstein s'est pendant un temps intéressé à la théorie des électrons dans les métaux, pensant trouver là le moyen de résoudre la difficulté liée à la juxtaposition, au sein de la physique pensée comme unitaire, de deux types de théories : l'une portant sur du continu (l'électromagnétisme) et l'autre sur du discontinu (la mécanique et la statistique qui la complète). Einstein et Mileva ont passionnément admiré le livre de celui que l'on considère à l'heure actuelle comme le père de la théorie du solide : Drude. On savait déjà qu'Einstein avait été, très jeune, un remarquable statisticien; on ignorait qu'il eût été aussi ce qu'en termes modernes nous nommerions un physicien du solide. Ceci d'ailleurs ne doit pas étonner, rétrospectivement du moins : la physique du solide n'est-elle pas généralement considérée comme la fille aînée de la théorie quantique ? Il suffit de renverser cet ordre de filiation pour concevoir qu'Einstein ait pu " flairer » dans la physique du solide la nécessité de la théorie quantique encore à naître.

La lecture de ces lettres ne laisse en tout cas aucun doute sur le rôle plus que catalytique joué par Mileva dans le développement intellectuel du jeune Einstein : "Notre recherche", dit-il lui-même à propos de sa tentative pour se passer de l'éther et constituer une véritable électrodynamique. Les historiens des sciences se sont toujours étonnés, et émerveillés, de ce que dans son article sur la relativité restreinte, Einstein n'ait cité aucun nom, si ce n'est celui de son ami Besso qu'il remercie à la dernière phrase pour les « discussions fructueuses " qu'il a eues avec lui. On peut imaginer, aujourd'hui que l'on connait les termes de leur collaboration, que si Einstein a agi de la sorte c'est parce qu'il lui aurait fallu associer sa propre femme à ses travaux; ce qui probablement ne "devait pas se faire". A preuve, le fait qu'à plusieurs reprises, Einstein parlant d'un article sur la capillarité, qu'il avait publié en 1901, dise " notre mémoire ", " notre travail ", etc., sans que Mileva trouve à redire au fait que « notre travail" paraisse sous son seul nom à lui.

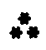

Qu'au bout de plusieurs années de recherches, une équipe produise des 
documents inédits jetant un nouvel éclairage sur un point d'histoire n'a, après tout, rien qui doive étonner. Plus surprenant est le fait que l'on commence seulement maintenant à lire et à prendre au sérieux la correspondance (non publiée, mais d'accès facile puisqu'elle se trouve dans les Archives Einstein à Boston, Princeton et Jérusalem) entre Einstein et Schrödinger : la stature même des deux protagonistes laisse supposer a priori que cet échange de lettres ne peut pas être sans intérêt. Et de fait, il s'avère que l'un des sujets en discussion entre Einstein et Schrödinger fut, aux environs de 1935, ce qu'il est convenu d'appeler " l'argument EPR » (le sigle EPR devant être entendu comme EinsteinPodolsky-Rosen, du nom des trois auteurs d'un article fameux paru en anglais en $1935^{1}$ ). Or s'il est un thème qui a alimenté les controverses et les débats ces derniers temps, c'est bien l'article EPR: depuis dix ans, physiciens, philosophes et physiciens-philosophes glosent abondamment sur le sujet. Pour parler vite et de façon schématique, on peut dire que l'argument EPR porte sur une expérience de pensée, relative à la mesure des grandeurs physiques, destinée à montrer que la théorie quantique, dans sa formulation et son interprétation canoniques, n'est pas « complète ", c'est-à-dire - toujours pour aller vite - n'établit pas une correspondance bi-univoque entre des « éléments de réalité " et les concepts de la théorie. Le cœur du débat est finalement bien résumé par le titre même de l'article EPR: "Can Quantum Mechanical Description of Physical Reality Be Considered Complete? " C'est évidemment le mot "réalité " (lequel, pour ne pas simplifier les choses, correspond en allemand à la fois à "Realität» et à "Wirklichkeit ») qui est à l'origine du flot de commentaires mentionnés il y a un instant. Chacun ici tire la couverture (entendez : l'autorité du "plus grand physicien de ce siècle ") à soi, les uns pour étayer une théorie "à variables cachées » qui tente désespérément de rendre classique la théorie quantique, les autres pour faire d'Einstein le porte-drapeau d'une position philosophique « réaliste ». Réalisme d'autant plus difficile à définir qu'Einstein s'est à plusieurs reprises défendu d'adhérer à telle ou telle position philosophique et s'est toujours considéré comme un opportuniste éclectique en cette matière. Il est donc étonnant, dans ces conditions que la correspondance qu'a entretenue Einstein avec Schrödinger dans les mois qui ont suivi la publication de l'article EPR n'ait pas plus retenu l'attention des commentateurs. Deux chercheurs, A. Fine et D. Howard (voir la bibliographie), ont ces dernières années corrigé cet "oubli ».

Tout commence par une lettre datée du 7 juin 1935 où Schrödinger, après avoir félicité Einstein d'avoir dans sa dernière publication (EPR) réveillé la théorie quantique endormie dans son dogmatisme, réclame néanmoins des éclaircissements sur le fond du problème. A quoi Einstein répond (le 19 juin) que, pour des raisons de langue (Einstein a toujours prétendu ne pas maîtriser l'anglais), c'est Podolsky qui a écrit l'article après qu'ils en eussent discuté ensemble. Mais, ajoute Einstein, l'argument est mal " sorti ", en tout cas pas comme lui l'aurait souhaité : le fond du problème est, dit-il, masqué sous un excès d'érudition.

1. A. Einstein, B. Podolsky, N. Rosen, «Can Quantum Mechanical Description of Physical Reality Be Considered Complete?», Physical Review, 47, 1935, p. 777-780. 
On en reste pantois; surtout si l'on songe à l'abondante littérature où l'argument EPR est invoqué comme étant l'expression même de la pensée d'Einstein.

Suit alors, toujours dans la même lettre, l'exposé de l'argument tel qu'Einstein le conçoit... et l'énonce de façon parfaitement claire (soit qu'il ait entre-temps affiné sa perception des choses, soit que l'usage de la langue allemande lui permette d'exprimer de façon plus nette la difficulté telle qu'il la perçoit). Considère, écrit-il en substance à Schrödinger, deux boîtes et une seule balle pouvant se trouver soit dans l'une, soit dans l'autre. Ce que, précise-t-il, on peut " observer » en soulevant le couvercle de l'une des boîtes. Dira-t-on que l'affirmation selon laquelle la balle a une probabilité $1 / 2$ de se trouver dans la première boîte constitue une description complète de l'état des choses? La réponse est non, si l'on adopte le point de vue de Bohr, selon lequel une description n'est complète que si l'on est en mesure de dire que la balle se trouve dans telle boîte. Mais ajoute Einstein, la réponse sera oui, si l'on adopte le point de vue de Schrödinger; en effet, dans cette conception, l'état de la première boîte est entièrement défini par la donnée de la probabilité $1 / 2$. Alors, que conclure : oui ou non? Commentaire d'Einstein : le philosophe talmudiste (on comprend par ailleurs qu'il s'agit de Bohr) dira que ces deux conclusions sont identiques et ne diffèrent que par leur mode d'expression (allusion, un peu sommaire, au principe de complémentarité). Tel n'est pas le point de vue d'Einstein. Au contraire, affirme-t-il, il faut répondre à la question par oui ou par non. Mais ajoute-t-il immédiatement, cela n'est possible qu'en introduisant un nouveau principe (plus satisfaisant que le principe de complémentarité), le principe de séparation (Trennungsprinzip), quelquefois traduit par principe de séparabilité, qui dit que ce qui se passe dans la deuxième boîte ne doit pas dépendre de ce qui se passe dans la première. Si l'on adhère à ce principe (et pour Einstein, c'est visiblement là que se situe le cceur de l'argumentation), alors il devient possible de répondre à la question posée. En effet, on remarque d'abord que le point de vue de Schrödinger devient inacceptable : ce point de vue est en contradiction avec le principe de séparation puisque l'état de la seconde boîte (autrement dit la probabilité d'y trouver la balle) dépend du fait qu'il existe une première boîte, laquelle accapare en quelque sorte une partie de la probabilité totale. Seul reste acceptable alors le point de vue de Bohr, d'après lequel - on l'a vu - la description donnée (en terme de probabilités n'est pas complète. Conclusion : la " théorie " correspondant à une description de l'état des boîtes par les probabilités est incomplète.

Mais ce qui intéresse Einstein, ce n'est pas cette «théorie " en tant que telle; celle-ci ne figure dans l'argumentation qu'à titre d'exemple destiné à illustrer une conclusion épistémologique, à savoir qu'une théorie doit satisfaire au principe de séparation pour pouvoir être dite complète.

Qu'en est-il dans ces conditions de cette " vraie " théorie qu'est la théorie quantique? Est-elle complète ou ne l'est-elle pas? Pour répondre à la question, il suffit, d'après ce qui vient d'être dit, d'examiner si elle satisfait au principe de séparation ou pas. Or, en théorie quantique, l'état d'un système est décrit par une fonction $\psi$. Cette description satisfait-elle au principe de séparation? Non, répond Einstein qui, à ce stade de son raisonnement et à ce stade seulement, introduit l'expérience de pensée qui est au centre de l'article EPR... en la 
dépouillant de ses complications inutiles, pour n'en garder que ce qui sert son propos. Cette expérience de pensée montre qu'il existe des systèmes pour lesquels la fonction $\psi$ dépend du type de mesure que l'on effectue sur un autre système (c'est le cas de deux systèmes ayant interagi à un instant antérieur, même si cette interaction a cessé depuis longtemps). Dès lors, la description du système par la fonction $\psi$ ne peut pas être dite complète, pas plus que la description de l'état des boîtes par les probabilités d'y trouver la balle, il y a un instant. Conclusion: la théorie quantique, dans sa forme actuelle (c'est-à-dire un formalisme dans lequel l'état du système est décrit par une fonction $\psi$ ) n'est pas complète pour la raison qu'elle ne satisfait pas au principe de séparation.

Les écarts entre cet argument reconstitué par Einstein pour répondre aux questions de Schrödinger et l'argument tel qu'il est paru dans l'article EPR ne sont pas mineurs, ils sautent aux yeux dès lors que l'on prend la peine de mettre les deux textes en regard.

Première différence, une différence de forme: d'un côté, un argument technique, savant, érudit ; de l'autre, une idée simple, développée d'abord à propos d'une «théorie " simplifiee, puis appliquée à la théorie quantique ellemême. Il ne faut pas croire qu'il ne s'agit là que d'une différence de style, car en physique, le style est primordial et un principe zéro de jugement en matière de théorie consiste à privilégier les idées simples par rapport aux développements techniques. Les idées simples ont, a priori, une plus grande valeur épistémologique que les arguments techniques; ne serait-ce que parce que l'on est toujours tenté de penser qu'un argument technique cache nécessairement une idée simple; ou, plus exactement, qu'il n'est pas d'argument technique valable qui ne puisse s'exprimer de façon "simple", la démarche théorique ne pouvant être considérée comme menée à son terme que si l'on a débusqué derrière la technicité l'idée simple qu'elle recouvre.

Deuxième différence: Einstein dans sa lettre à Schrödinger introduit un principe supplémentaire (celui de séparation) qui ne figure pas dans l'article EPR. Il semble dès lors que toute discussion concernant le " réalisme " d'Einstein ne puisse faire l'économie de l'analyse de ce principe, lequel a d'ailleurs subi plusieurs modifications dans les écrits suivants d'Einstein.

Troisième différence : la définition de la complétude donnée par Einstein dans sa correspondance avec Schrödinger ne recouvre pas exactement celle donnée dans l'article EPR. Dans ce dernier, en effet, une théorie est dite complète "si tout élément de réalité physique a une contrepartie dans la théorie physique ", alors que dans sa lettre du 19 juin 1935 Einstein pose qu'une théorie ne décrit de façon complète la réalité (Wirklichkeit) que si la fonction $\psi$ qui décrit l'état du système est liée de façon bi-univoque à l'état réel (wirklich, encore) du système réel (wirklich). On voit que la condition exigée par Einstein est plus restrictive que celle formulée par l'article EPR. Cette dernière, en effet, porte sur l'état du système, alors que la condition exigée par Einstein porte sur la fonction $\psi$ qui représente l'état du système. Or il se trouve qu'en théorie quantique, à un même état du système correspondent plusieurs fonctions le représentant, différant entre elles par un facteur (imaginaire pur) de phase ; on conçoit donc l'importance de cette différence liée au mode même de représentation inauguré par la théorie quantique. En somme, comme le souligne très justement $D$. 
Howard, la condition de complétude exprimée dans l'article EPR ne contient que la moitié de celle avancée par Einstein.

En définitive, ce que passe sous silence l'article EPR, c'est la difficile (mais combien riche) question de la phase, cette partie « imaginaire " des concepts mathématico-physiques aux effets pourtant bien «réels ». En ce sens, Einstein a parfaitement choisi son expression pour manifester sa désapprobation : l'argument est mal «sorti ». Tel un mauvais cliché photographique, l'article EPR ne fait pas ressortir l'essentiel qui reste noyé dans un fonds uniforme de difficultés techniques sans importance. Plus même : en centrant son analyse sur la mesure de deux grandeurs " incompatibles " (la position et la quantité de mouvement dont on sait qu'elles ne peuvent être mesurées avec précision simultanément, comme l'indiquent les relations de Heisenberg), Podolsky a braqué les projecteurs sur un point (les relations de Heisenberg) sans importance dans toute cette affaire (comme l'indique très clairement Einstein dans sa lettre à Schrödinger). Podolsky laisse ainsi entendre que les difficultés de la théorie quantique tiennent à l'existence des relations de Heisenberg, alors que l'origine du caractère " incompréhensible " de la théorie se situe bien en amont, dans la nature même de la représentation mathématique qui la constitue.

\section{**}

C'est à dessein que j'ai centré cette revue des études einsteiniennes sur deux points particuliers : pour bien montrer l'état fragmentaire dans lequel elles se trouvent. C'est à regret que je me suis résolue à revenir une fois de plus sur cette tarte à la crème des commentaires «philosophiques » concernant l'œuvre d'Einstein qu'est l'argument EPR. Mais il le fallait, pour montrer à quel point, même dans ce domaine archirebattu, il reste à faire : la position réelle d'Einstein reste largement ignorée.

C'est pourquoi toute étude prétendant exposer les vues épistémologiques et philosophiques d'Einstein est à mon avis prématurée. Le fait est que, depuis vingt ans, ce sont toujours les mêmes textes (essentiellement ceux qui ont été traduits en anglais, sans d'ailleurs que cette traduction soit entièrement fiable) qui alimentent ce genre de considérations globales. Il faut absolument sortir de ce confinement intellectuel; il faut que l'admirable travail d'Helen Dukas n'ait pas été accompli en vain. Il faut découvrir le véritable Einstein, tel qu'il se trouve dans ses archives.

C'est dans cette optique que le C.N.R.S. a mis en chantier une édition d'œuvres choisies d'Einstein en français (si les traductions d'Einstein en anglais laissent parfois à désirer, celles en français actuellement disponibles sont franchement désastreuses). Cette publication (co-édition Le Seuil-C.N.R.S.), dont les premiers volumes doivent être disponibles au début de l'année 1989, comportera six volumes: Théorie quantique et Mécanique statistique, deux volumes portant sur les théories de la relativité et la Cosmologie, un volume d'Écrits politiques, un d'Écrits philosophiques et épistémologiques, un volume de Correspondance avec des personnes (françaises ou non) résidant en France. Dans les trois premiers volumes, à caractère plus technique que les trois autres, on trouvera des articles 
scientifiques (en nombre restreint), des écrits de vulgarisation, et surtout de nombreuses lettres (l'analyse de la correspondance Einstein-Schrödinger commentée plus haut laisse entrevoir à quel point la connaissance de ce qu'Einstein exprimait en privé est importante). En promouvant cette publication, le C.N.R.S. entend donner aux chercheurs les moyens d'étude qui leur manquent actuellement; en choisissant une formule à large diffusion, il entend aussi rectifier l'image que dans le "grand public" on se fait d'Einstein.

Françoise BALIBAR, Université de Paris VII, RCP du C.N.R.S. "Édition d'Einstein en francais".

\section{QUELQUES INDICATIONS BIBLIOGRAPHIQUES (LIMITEES AUX HUIT DERNIËRES ANNEES)}

\section{L'ÉDITION DE RÉFÉRENCE :}

The Collected Papers of Albert Einstein. Vol. I : The Early Years, 1879-1902, ed. John Stachel, Princeton, Princeton University Press, 1987.

OUVRAGES REGROUPANT DES CONTRIBUTIONS ÉCRITES À L'OCCASION DU CENTENAIRE DE LA NAISSANCE D'EINSTEIN (1979):

Some Strangeness in the Proportion, ed. Harry Woolf, Reading, Mass., AddisonWesley, 1980.

Einstein Symposium Berlin, ed. H. Nelkowski, A. HermanN, H. Poser, R. SCHAder, R. SEILER, Berlin, Springer Verlag, 1979.

Albert Einstein. Historical and Cultural Perspectives. The Centennial Symposium in Jerusalem, ed. by Gerald Holton, Yehuda Elkana, Princeton, Princeton University Press, 1982.

After Einstein, ed. Peter Barker, Cecil G. Shugart, Memphis, TN, Memphis State University Press, 1981. 
DEUX OUVRAGES FONDAMENTAUX POUR L'ÉTUDE DE L'CEUVRE SCIENTIFIQUE :

Arthur I. Miller, Albert Einstein's Special Theory of Relativity, Emergence (1905) and Early Interpretation (1905-1911), Reading, Mass., Addison-Wesley, 1981. Abraham PAIS, Subtle is the Lord... The Science and Life of Albert Einstein, Oxford/New York, Oxford University Press, 1982.

QUELQUES RECUEILS D'ARTICLES RÉCEMMENT PARUS :

Athur FINE, The Shaky Game. Einstein Realism and the Quantum Theory, Chicago, The University of Chicago Press, 1986.

L. PyENSON, The Young Einstein. The Advent of Relativity, Bristol, Adam Hilger Ltd, 1985.

Gerald Holton, The Advancement of Science and its Burdens, The Jefferson Lectures and Other Essays (dont la moitié sont consacrés à Einstein), New York/Cambridge, Cambridge University Press, 1987.

À PROPOS DE EPR (quelques-unes des dernières publications) :

J. JARRETT, "On the Physical Significance of the Locality Conditions in the Bell Arguments ", Noûs, 1984.

Don HowarD, "Einstein on Locality and Separability ", Studies in History and Philosophy of Science, 16, 3, 1985, p. 171-201.

L'édition française d'Euvres choisies d'Albert Einstein (6 vols) est publiée conjointement par les Éditions du Seuil et du C.N.R.S. Les deux premiers volumes (Correspondance française et Quanta) paraîtront au printemps 1989. 